\title{
Enfermedad de Graves y cirugía. Análisis de 49 pacientes operados en el período 1995-2002 en el Hospital Clínico Regional de Valdivia
}

\author{
Juan Antonio Pérez P, Sebastián Soto G, Constanza Rudolph Q*.
}

\section{RESUMEN}

La enfermedad de Graves es una patología autoinmune que afecta la glándula tiroides de tal forma de aumentar su función, caracterizándose clásicamente por bocio difuso, oftalmopatía y taquicardia. Afecta con mayor frecuencia al sexo femenino entre la $3^{a}$ y $4^{a}$ décadas de vida con una relación mujer:hombre de 7:1 en áreas bociógenas, siendo inferior en áreas no bociógenas. Se presentan las características clínicas y los resultados de la cirugía en 49 pacientes operados entre Enero de 1995 y Diciembre del 2002 en el Servicio de Cirugía del Hospital Clínico Regional de Valdivia. Cuarenta y un pacientes fueron del sexo femenino y 8 del masculino, con una relación 5:1. La edad media de la serie fue de 30 años, fluctuando entre 8 y 61 años. El motivo de consulta más frecuente fue el aumento de volumen tiroideo asociado a síntomas y/o signos de hipertiroidismo en 26 pacientes (54\%). El tiempo promedio entre la primera consulta a endocrinología y el traslado a cirugía fue de 13,6 meses, variando entre 1 y 69 meses. La cirugía de elección fue la tiroidectomía subtotal en 47 pacientes. La complicación postoperatoria más frecuente fue la hipocalcemia transitoria que ocurrió en 5 casos. En el estudio histopatológico todos los especímenes operatorios resultaron compatibles con enfermedad de Graves, destacando un caso incidental de cáncer papilar. Los pacientes fueron dados de alta en promedio al $4^{\circ}$ día postoperatorio, rango entre $2^{\circ}$ y $5^{\circ}$ día. En la serie no hubo mortalidad. (Palabras claves/Key words: Hipertiroidismo/Hyperthyroidism; Enfermedad de Graves/Graves disease, Tratamiento/Treatment, Cirugía/Surgery).

\section{INTRODUCCIÓN}

La enfermedad de Graves, de Parry o Basedow son distintos nombres utilizados para definir un trastorno autoinmune que afecta a la tiroides, mediante la producción de autoanticuerpos anti receptores de TSH en la superficie de la glándula (TRAb) y a través de ella a otros órganos y sistemas ${ }^{1}$.

Aunque fue descrita por el galeno Irlandés Robert James Graves en 1834, los síntomas y signos de ella datan de muchos años e incluso siglos atrás, tal como el exoftalmo mencionado por Aristóteles y Xenophon en el siglo $\mathrm{V}$ a.C. ${ }^{2}$.
Las primeras descripciones en la era moderna datan de 1786, año en el cual el médico inglés Caleb Parry, atendió una paciente de sexo femenino de 37 años, con taquicardia, exoftalmo y bocio, causándole tal impresión que describió: "Los ojos protruían de sus órbitas... y en cada sístole el corazón hacía temblar todo su cuerpo",2, . Durante su vida conoció a 4 enfermas más de similares características, sin llegar a asociarlas entre sí. Tres años luego de su muerte, en 1825 su hijo publicó estos casos ${ }^{2}$.

Posteriormente con 5 años de diferencia, Robert James Graves en Dublín, Irlanda y Carl Adolph von Basedow en Mersebury, Alemania, 
engloban todas las manifestaciones dentro de un solo síndrome, en los años 1835 y 1840 respectivamente ${ }^{3}$.

Esta patología afecta principalmente al sexo femenino en la $3^{\mathrm{a}}$ o $4^{\mathrm{a}}$ década de la vida, con una relación mujer:hombre de 7:1 en áreas bociógenas, la que desciende en áreas no bociógenas. Se han diferenciado múltiples haplotipos según la raza de la persona afectada, como también el carácter familiar de la enfermedad ${ }^{1}$.

El manejo de la enfermedad de Graves es fundamentalmente médico, sin embargo existen ciertas condiciones, que se enumeran en la Tabla 1, en que tiene lugar el tratamiento quirúrgico.

Tabla 1. Indicaciones de tratamiento quirúrgico ${ }^{3}$.

- Enfermedad de Graves con nódulo sospechoso de cáncer de tiroides.

- Bocio voluminoso con fracaso a tratamiento médico.

- Niños que no toleran, no se adaptan o recidivan con el tratamiento con medicamentos antitiroideos.

- Pacientes embarazadas que no responden al tratamiento médico o son alérgicas a éste.

- Pacientes que rechazan o manifiestan temor al tratamiento con radioyodo.

- Enfermas con síntomas de compresión de la vía aereodigestiva superior.

A pesar que las indicaciones anteriores están bien establecidas, el manejo quirúrgico de esta patología ha sido bastante controversial a lo largo del tiempo, dado principalmente por la morbimortalidad a que se expone al paciente y por la elección de una técnica quirúrgica que proporcione los mejores resultados y las menores complicaciones.

En atención a estas consideraciones decidimos estudiar a 49 pacientes portadores de enfermedad de Graves operados en el Servicio de Cirugía del Hospital Clínico Regional de Valdivia, en un período de 8 años, con el fin de establecer las características clínicas que presentó la enfermedad y evaluar los resultados obtenidos con el tratamiento quirúrgico.

\section{MATERIAL Y MÉTODOS}

Se analizaron en forma retrospectiva las fichas clínicas y estudio histopatológico de 49 pacientes portadores de enfermedad de Graves, operados entre Enero de 1995 y Diciembre del año 2002.

Los datos fueron ingresados a una planilla Microsoft Excel, incluyéndose los siguientes parámetros: edad, sexo, tiempo entre la primera consulta y derivación para cirugía, motivo de consulta, patologías asociadas, antecedentes familiares, indicación quirúrgica, función tiroidea pre cirugía, tipo de cirugía, complicaciones post operatorias, estudio histopatológico y tiempo de hospitalización.

El análisis estadístico se realizó utilizando las opciones del programa computacional antes mencionado.

La preparación preoperatoria de estos pacientes consistió en lugol 15 gotas cada 8 horas más propanolol $40 \mathrm{mg}$ cada 8 horas durante 2 semanas.

\section{RESULTADOS}

La serie comprendió 49 pacientes, 41 $(83,6 \%)$ del sexo femenino y $8(16,4 \%)$ de sexo masculino. La edad media fue de 30 años, fluctuando entre 8 y 61 años, incluyendo 4 niños entre 8 y 13 años.

La relación entre ambos sexos fue de 5:1 (mujer:hombre).

El tiempo promedio entre la primera consulta a endocrinología y la derivación a cirugía fue de 13,61 meses, con un rango entre 1 y 69 meses.

El motivo principal de consulta fue el bocio difuso asociado a síntomas y/o signos de hipertiroidismo en 26 (54\%) casos. Las causas de consulta se desglosan en la Tabla 2.

Ocho pacientes presentaban patologías asociadas, 5 de ellos diabetes mellitus y 3 hipertensión arterial.

Destaca en la serie 9 pacientes con familiares directos portadores de enfermedad de Graves, cuyo grado de parentesco se muestra en la Tabla 3.

La indicación quirúrgica más frecuente fue el fracaso al tratamiento médico, destacando el caso de una embarazada de 13 semanas alérgica al PTU que se operó exitosamente durante el segundo trimestre de embarazo. Las indicaciones de tratamiento quirúrgico de los pacientes de la serie se muestran en la Tabla 4. 
Tabla 2. Motivo de consulta.

\begin{tabular}{|lcc|}
\hline Motivo de consulta & No $^{\circ}$ casos & Porcentaje (\%) \\
\hline Bocio difuso más signos y/o síntomas de hipertiroidismo & 26 & 54 \\
Síntomas y/o signos de hipertiroidismo & 11 & 22 \\
Bocio difuso & 8 & 16 \\
Exoftalmo & 2 & 4 \\
Disnea & 2 & 4 \\
\hline
\end{tabular}

Tabla 3. Familiares portadores de enfermedad de Graves.

\begin{tabular}{|lc|}
\hline Familiares afectados & N $^{\circ}$ casos \\
\hline Dos hermanos & 1 \\
Tía materna & 1 \\
Hermano & 2 \\
Padre & 2 \\
Abuelo paterno y tío paterno & 1 \\
Madre & 1 \\
Abuela materna e hija. & 1 \\
\hline
\end{tabular}

Tabla 4. Indicaciones de tratamiento quirúrgico.

\begin{tabular}{|lcc|}
\hline Indicación de cirugía & $\begin{array}{c}\text { No } \\
\text { casos }\end{array}$ & $\begin{array}{c}\text { Porcentaje } \\
(\%)\end{array}$ \\
\hline Fracaso tratamiento médico & 38 & 78 \\
Estética & 5 & 10 \\
Alergia a propiltiouracilo & 3 & 6 \\
Síntomas compresivos & 2 & 4 \\
Nódulo tiroideo predominante & 1 & 2 \\
\hline
\end{tabular}

Trece pacientes (27\%) lograron el eutiroidismo completo previo a la cirugía, los 36 restantes $(73 \%)$ presentaban algún signo de hiperfunción tiroidea.

En 23 (47\%) casos se realizó laringoscopía indirecta pre cirugía, encontrándose normal en todos ellos.

La estimación clínica del peso de la glándula tiroides fluctuó entre 40 y 100 g, con una media de $80 \mathrm{~g}$.

La cirugía de elección en el $96 \%$ de los pacientes (47 casos) fue la tiroidectomía subtotal; en los 2 restantes se realizó una tiroidectomía total y una lobectomía más istmectomía respectivamente.

En todos los casos, la irrigación de la glándula estaba aumentada, las glándulas paratiroides fueron visualizadas en 42 casos (86\%). El nervio recurrente laríngeo fue visualizado a ambos lados en 27 casos (55\%), en los 22 restantes (45\%) no se visualizó.

En los casos de tiroidectomía subtotal el peso estimado del remanente fluctuó entre 2 y $6 \mathrm{~g}$ con una media de $4 \mathrm{~g}$.

El sangrado estimado en el intraoperatorio fluctuó entre 10 y 200 cc con una media de 40 cc.

El tiempo operatorio medio fue de 125 minutos con un rango entre 85 y 210 minutos.

En todos los casos se realizó laringoscopía directa al extubar al paciente, cuyos resultados se exponen en la Tabla 5.

Tabla 5. Laringoscopía directa postoperatoria.

\begin{tabular}{|lcc|}
\hline Resultado & No $^{\circ}$ casos & Porcentaje (\%) \\
\hline Paresia cuerda vocal izquierda & 1 & 2 \\
Paresia cuerda vocal derecha & 3 & 6 \\
Normal & 45 & 92 \\
\hline
\end{tabular}


Es importante recalcar que ningún caso con paresia de cuerda vocal presentó voz bitonal una vez recuperado de la anestesia.

La complicación más frecuente fue la hipocalcemia en 5 (10\%) casos, la cual se manejó con gluconato de calcio endovenoso y luego calcio oral. Destacan además 2 (4\%) casos de hematomas cervicales no compresivos que no requirieron una reoperación y que se manejaron con punción en la sala a partir de las $36 \mathrm{~h}$ post cirugía. Las complicaciones postoperatorias se enumeran en la Tabla 6 .

Tabla 6. Complicaciones postoperatorias.

\begin{tabular}{|lcc|}
\hline Complicaciones & $\begin{array}{c}\mathbf{N}^{\mathbf{0}} \\
\text { casos }\end{array}$ & $\begin{array}{c}\text { Porcentaje } \\
\text { (\%) }\end{array}$ \\
\hline Hipocalcemia & 5 & 10 \\
Hematoma cervical & 2 & 4 \\
Bronquitis purulenta & 1 & 2 \\
Sin complicaciones & 40 & 84 \\
\hline
\end{tabular}

No hubo mortalidad perioperatoria.

Al examen histopatológico el peso de la glándula fluctuó entre 25 y $170 \mathrm{~g}$ con una media de $70 \mathrm{~g}$. El estudio de la pieza confirmó en todos los casos la enfermedad de Graves encontrando un caso incidental de carcinoma papilar, en forma de un nódulo intraparenquimatoso de $4 \mathrm{~mm}$ de diámetro alejado de la cápsula.

El tiempo de hospitalización medio fue de 4 días, con un rango entre 2 y 5 días. Un paciente debió ser trasladado al servicio de medicina para manejo de descompensación metabólica de diabetes mellitus.

En los 36 pacientes que presentaron algún grado de hiperfunción tiroidea durante el preoperatorio, ésta se controló con propanolol, regresando entre el $4^{\circ}$ y $16^{\circ}$ día postoperatorio con una mediana de 8 días.

\section{DISCUSIÓN}

La cirugía como opción de tratamiento de la enfermedad de Graves tiene indicaciones precisas, las cuales fueron previamente mencionadas en la Tabla 1. Actualmente se acepta como una terapia válida y vigente junto al resto de alternativas terapéuticas que se utilizan con mayor frecuencia y que incluyen el tratamiento farmacológico, el radioyodo y últimamente la embolización selectiva de las arterias tiroideas superiores e inferiores ${ }^{2-4}$.

El requisito fundamental para validar la cirugía es que logre un rápido control del hipertiroidismo y que presente un porcentaje de morbimortalidad aceptable de acuerdo a los cánones internacionales.

El tratamiento quirúrgico de la enfermedad de Graves fue duramente cuestionado en sus albores debido a su alta morbimortalidad, al punto que aquellos que lo realizaban fueron calificados por Samuel Gross, en el año 1850, como carniceros y el procedimiento en sí como una carnicería ${ }^{3}$.

A pesar de lo anterior, Kocher publicó en 1883 el resultado obtenido en 101 tiroidectomías, con una mortalidad operatoria de $12,8 \%$. Tal fue el impacto que causó, que tuvo el honor de ser el primer cirujano en recibir el premio Nobel de Medicina. Al momento de su muerte había practicado más de 5000 tiroidectomías, reduciendo la mortalidad operatoria a menos del $1 \%$, cifras que aún están vigentes ${ }^{3,5-8}$.

En cuanto a nuestra serie, vemos un fiel reflejo de los parámetros epidemiológicos publicados internacionalmente ${ }^{1-3}$.

Es interesante el hecho de que 9 pacientes de la serie tengan antecedentes familiares de enfermedad de Graves, lo que sirve para certificar el carácter familiar de esta patología $a^{1-3}$.

Destaca en la serie una paciente con un embarazo de 13 semanas, que representa el $2 \%$ de la serie, algo superior al $0,75 \%$ publicado internacionalmente ${ }^{3}$. En el caso de embarazadas es conveniente recordar que la cirugía se lleva a cabo durante el $2^{\circ}$ trimestre y que el diagnóstico es difícil dado los cambios fisiológicos del embarazo que llevan a un aumento de los niveles de $T_{3}$ y $T_{4}$ séricos.

En nuestra serie la cirugía de elección fue la tiroidectomía subtotal total (TST), dejando un remanente promedio de glándula de $4 \mathrm{~g}$. La preferencia de esta técnica se basa en su menor tasa de complicaciones, tal como hipocalcemia y lesión del nervio laríngeo recurrente, pero por sobre todo, por su supuesta baja incidencia de hipotiroidismo a largo plazo, la que pretende evitar en al menos $50 \%$ la terapia de reemplazo con hormona tiroidea, propósito que actualmente está en evaluación. A pesar de lo anterior, estudios internacionales han demostrado que la cirugía de elección ha de ser la tiroidectomía total (TT), ya que permite tener un resultado predecible en forma casi inmediata de la función tiroidea. Además estos 
mismos estudios han demostrado que el $70-80 \%$ de los pacientes sometidos a TST se harán hipotiroideos dentro del año de la cirugía ${ }^{3,5-8}$. A pesar de estos hechos, creemos que con la TST hemos logrado el objetivo fundamental del tratamiento de la enfermedad de Graves que consiste en rápido control de los signos y síntomas de la enfermedad, recuperar y mantener el eutiroidismo, baja morbilidad y mortalidad y un costo razonable ${ }^{3}$.

La asociación entre cáncer y enfermedad de Graves no es despreciable y en distintas series fluctúa entre 5 y $10 \%$, , 10 . Aquellos casos en que el cáncer es menor a $1 \mathrm{~cm}$, su genio biológico no varía, no así cuando supera este tamaño, caso en el cual se torna más agresivo ${ }^{3,9,10}$.

Por otro lado, en el intraoperatorio es recomendable la visualización de los nervios laríngeos recurrentes y las paratiroides con el fin de evitar iatrogenias, en aquellos casos en que se opte por TT, de no ser posible se recomienda disección cuidadosa muy cercana a la glándula.
A pesar de la óptima visualización de las paratiroides hay casos de hipocalcemia postoperatoria, dada por el traumatismo de la zona e isquemia transitoria. Con el fin de evitar lo anterior, algunos autores proponen el autotrasplante de estas glándulas ${ }^{11,12}$.

Debe existir especial cuidado en preservar los nervios laríngeos recurrentes, ya que su lesión se traduce en una secuela importante. A pesar de su visualización, la lesión de ellos fluctúa entre 1-3\% ${ }^{13-16}$. En nuestra serie hubo 4 casos de paresia de cuerdas vocales a pesar de haber visualizado los nervios sólo en 27 casos.

El análisis de los resultados de nuestra serie nos corrobora que la cirugía es un arma terapéutica válida en casos seleccionados de pacientes portadores de enfermedad de Graves, ya que logra controlar el hipertiroidismo en forma rápida y eficaz, mediante una técnica quirúrgica que conlleva un número de complicaciones postoperatorias razonables y manejables.

\section{REFERENCIAS}

1. Wartofsky L. Enfermedades del tiroides. En: Harrison, Principios de Medicina Interna. Madrid, McGraw-Hill, 1994: 2238-46

2. Sawin CT: Theories of causation of Graves' disease. A historical perspective. Endocrinol Metab Clin North Am 1998; 27: 63-72

3. Alsanea O, Clark OH: Treatment of Graves' disease: the advantages of surgery. Endocrinol Metab Clin North Am 2000; 29: 321-37

4. Xiao H, Zhuang W, Wang S, Yu B, Chen G, et al: Arterial embolization: a novel approach to thyroid ablative therapy for Graves' disease. J Clin Endocrinol Metab 2002; 87: 3583-9

5. Barakate MS, Agarwal G, Reeve TS, Barraclough B, Robinson B, Delbridge LW: Total thyroidectomy is now the preferred option for the surgical management of Graves' disease. ANZ J Surg 2002; 72: 321-4

6. Sánchez J, Lamata F, García F, et al. Elección de la técnica quirúrgica en el tratamiento de la enfermedad de Graves-Basedow. Cirujano General 2000; 22: 311-8

7. Thomusch O, Machens A, Sekulla C, Ukkat $\mathrm{J}$, Brauckhoff $\mathrm{M}$, Dralle $\mathrm{H}$ : The impact of surgical technique on postoperative hypoparathyroidism in bilateral thyroid surgery: a multivariate analysis of 5846 consecutive patients. Surgery 2003; 133: 180-5

8. Liu Q, Djuricin G, Prinz RA: Total thyroidectomy for benign thyroid disease. Surgery 1998; 123: 2-7
9. Pellegriti G, Belfiore A, Giuffrida D, Lupo L, Vigneri R: Outcome of differentiated thyroid cancer in Graves' patients. J Clin Endocrinol Metab 1998; 83: 2805-9

10. Kraimps JL, Bouin-Pineau MH, Mathonnet M, De Calan L, Ronceray J, et al: Multicentre study of thyroid nodules in patients with Graves' disease. Br J Surg 2000; 87: 1111-3

11. Lo CY, Lam KY: Routine parathyroid autotransplantation during thyroidectomy. Surgery 2001; 129: 318-23

12. Lo CY, Lam KY: Postoperative hypocalcemia in patients who did or did not undergo parathyroid autotransplantation during thyroidectomy: a comparative study. Surgery 1998; 124: 1081-7

13. Moley JF, Lairmore TC, Doherty GM, Brunt LM, DeBenedetti MK: Preservation of the recurrent laryngeal nerves in thyroid and parathyroid reoperations. Surgery 1999; 126: 673-9

14. Wheeler $\mathrm{MH}$. Thyroid surgery and the recurrent laryngeal nerve. Br J Surg 1999; 86: 291-2

15. Lo CY, Kwok KF, Yuen PW: A prospective evaluation of recurrent laryngeal nerve paralysis during thyroidectomy. Arch Surg 2000; 135: 204-7

16. Hermann M, Alk G, Roka R, Glaser K, Freissmuth $\mathrm{M}$ : Laryngeal recurrent nerve injury in surgery for benign thyroid diseases: effect of nerve dissection and impact of individual surgeon in more than 27,000 nerves at risk. Ann Surg 2002; 235: 261-8 